\title{
The Meixner Polynomials in Several Variables
}

\section{Nejla Özmen ${ }^{1 *}$, Esra Erkus-Duman²}

1 Düzce University, Faculty of Art and Science, Department of Mathematics, Konuralp TR-81620, Düzce, Turkey.

${ }^{2}$ Gazi University, Faculty of Science, Department of Mathematics, Teknikokullar TR-06500, Ankara, Turkey.

* Corresponding author. Tel.: +903805421100; email: nejlaozmen06@gmail.com, eerkusduman@gmail.com Manuscript submitted February 12, 2019; accepted April 9, 2019.

doi: 10.17706/ijapm.2019.9.3.144-151

\begin{abstract}
The polynomials are applied in many areas of mathematics, for instance, continued fractions, operator theory, analytic functions, interpolation, approximation theory, numerical analysis, electrostatics, statistical quantum mechanics, special functions, number theory, combinatorics, stochastic processes. On the other hand, generating functions have a great importance in special functions theory. The present study deals with some new properties of the Meixner polynomials in several variables. Firstly, we obtained some results include various families of multilinear and multilateral generating functions for the Meixner polynomials in several variables. In the last section, we get a theorem that gives a bilateral generating functions for these polynomials and the Lauricella functions. Finally, we derive some corollaries of the last theorem.
\end{abstract}

Key words: Bilinear and bilateral generating function, Lauricella function, Meixner polynomials.

\section{Introduction}

The Meixner polynomials are denoted by $m_{n}(x ; \beta, c)$ and are defined as [1]

$$
\sum_{n=0}^{\infty} m_{n}(x ; \beta, c) \frac{t^{n}}{n !}=(1-t)^{-\beta-x}\left(1-\frac{t}{c}\right)^{x}
$$

where $\beta>0, \quad 0<c<1$ and $x=0,1,2, \ldots$.

It is from (1) that (see [1]):

$$
m_{n}(x ; \beta, c)=\sum_{k=0}^{n} \frac{(\beta)_{n}(-x)_{k} n !}{k !(n-k) !(\beta)_{k}}\left(\frac{1}{c}-1\right)^{k},
$$

where $(\lambda)_{v}$ denotes the Pochhammer symbol.

Definition 1: The Meixner polynomials of s-variables are defined by [2]:

$$
\begin{aligned}
& m_{n}\left(x_{1}, \ldots, x_{s} ; \beta, c_{1}, \ldots, c_{s}\right) \\
& =\sum_{r_{1}=0}^{n} \sum_{r_{2}=0}^{n-r_{1}} \ldots \sum_{r_{s}=0}^{n-r_{1} \ldots-r_{s-1}} \frac{(\beta)_{n}(-n)_{r_{1}+\ldots+r_{s}} \prod_{j=1}^{s}\left(-x_{j}\right)_{r_{j}} \prod_{j=1}^{s}\left(1-c_{j}^{-1}\right)^{r_{j}}}{(\beta)_{r_{1}+\ldots+r_{s}} \prod_{j=1}^{s}\left(r_{j}\right) !},
\end{aligned}
$$


where $\beta>0, \quad 0<c_{j}<1 \quad x_{j}=\quad, 1$. and $j=1,2, \ldots, s$.

Theorem 1: (see [2]) The following generating functions holds true for the Meixner polynomials of s-variables defined by (2):

$$
\begin{gathered}
\sum_{n=0}^{\infty} m_{n}\left(x_{1}, \ldots, x_{s} ; \beta, c_{1}, \ldots, c_{s}\right) \frac{t^{n}}{n !}=(1-t)^{-\beta-x_{1}-\ldots-x_{s}} \prod_{j=1}^{s}\left(1-\frac{t}{c_{j}}\right)^{x_{j}} \\
\left(|t|<\min \left\{1,\left|c_{1}\right|, \ldots,\left|c_{s}\right|\right\}\right) .
\end{gathered}
$$

Theorem 2: (see [2]) The following generating function relationship for the Meixner polynomials of $s$ variables holds true:

$$
\begin{aligned}
& \sum_{n=0}^{\infty} m_{n+k}\left(x_{1}, \ldots, x_{s} ; \beta, c_{1}, \ldots, c_{s}\right) \frac{t^{n}}{n !} \\
& =(1-t)^{-\beta-k-x_{1}-\ldots-x_{s}} \prod_{j=1}^{s}\left(1-\frac{t}{c_{j}}\right)^{x_{j}} m_{k}\left(x_{1}, \ldots, x_{s} ; \beta, \frac{c_{1}-t}{1-t}, \ldots, \frac{c_{s}-t}{1-t}\right) .
\end{aligned}
$$

In Section 2, we establish several theorems involving various families of generating functions for the Meixner polynomials $m_{n}\left(x_{1}, \ldots, x_{s} ; \beta, c_{1}, \ldots, c_{s}\right)$ by applying the method which was discussed by Chen and Srivastava [3]. In the last section, we derive several families of bilateral generating functions for the multivariable Meixner polynomials and the generalized Lauricella functions.

\section{Bilinear and Bilateral Generating Functions}

In this section, we derive several bilinear and bilateral generating functions for the Meixner polynomials $m_{n}\left(x_{1}, \ldots, x_{s} ; \beta, c_{1}, \ldots, c_{s}\right)$ which generated by (2) and given explicitly by (3) using the similar method considered in [4]-[7].

Theorem 3: Let

$$
\begin{aligned}
& \Theta_{n, p}^{\mu, \psi}\left(x_{1}, \ldots, x_{s} ; \beta, c_{1}, \ldots, c_{s} ; y_{1}, \ldots, y_{r} ; \xi\right) \\
& :=\sum_{k=0}^{[n / p]} a_{k} m_{n-p k}\left(x_{1}, \ldots, x_{s} ; \beta, c_{1}, \ldots, c_{s}\right) \Omega_{\mu+\psi k}\left(y_{1}, \ldots, y_{r}\right) \frac{\xi^{k}}{(n-p k) !} .
\end{aligned}
$$

If

$$
\Lambda_{\mu, \psi}\left(y_{1}, \ldots, y_{r} ; \zeta\right):=\sum_{k=0}^{\infty} a_{k} \Omega_{\mu+\psi k}\left(y_{1}, \ldots, y_{r}\right) \zeta^{k}
$$

then, for every nonnegative integer $\mu$, we have

$$
\sum_{n=0}^{\infty} \Theta_{n, p}^{\mu, \psi}\left(x_{1}, \ldots, x_{s} ; \beta, c_{1}, \ldots, c_{s} ; y_{1}, \ldots, y_{r} ; \frac{\eta}{t^{p}}\right) t^{n}
$$




$$
=(1-t)^{-\beta-x_{1}-x_{2}-\ldots-x_{s}} \prod_{j=1}^{s}\left(1-\frac{t}{c_{j}}\right)^{x_{j}} \Lambda_{\mu, \psi}\left(y_{1}, \ldots, y_{r} ; \eta\right)
$$

Proof: If we denote the left-hand side of (6) by $T$ and use (5),

$$
T=\sum_{n=0}^{\infty} \sum_{k=0}^{[n / p]} a_{k} m_{n-p k}\left(x_{1}, \ldots, x_{s} ; \beta, c_{1}, \ldots, c_{s}\right) \Omega_{\mu+\psi k}\left(y_{1}, \ldots, y_{r}\right) \eta^{k} \frac{t^{n-p k}}{(n-p k) !} .
$$

Replacing $n$ by $n+p k$,

$$
\begin{array}{r}
T=\sum_{n=0}^{\infty} \sum_{k=0}^{\infty} a_{k} m_{n}\left(x_{1}, \ldots, x_{s} ; \beta, c_{1}, \ldots, c_{s}\right) \Omega_{\mu+\psi k}\left(y_{1}, \ldots, y_{r}\right) \eta^{k} \frac{t^{n}}{n !} \\
=\sum_{n=0}^{\infty} m_{n}\left(x_{1}, \ldots, x_{s} ; \beta, c_{1}, \ldots, c_{s}\right) \frac{t^{n}}{n !} \sum_{k=0}^{\infty} a_{k} \Omega_{\mu+\psi k}\left(y_{1}, \ldots, y_{r}\right) \eta^{k} \\
=(1-t)^{-\beta-x_{1}-x_{2}-\ldots-x_{s}} \prod_{j=1}^{s}\left(1-\frac{t}{c_{j}}\right)^{x_{j}} \Lambda_{\mu, \psi}\left(y_{1}, \ldots, y_{r} ; \eta\right)
\end{array}
$$

which completes the proof.

Theorem 4: For a non-vanishing function $\Omega_{\mu}\left(y_{1}, \ldots, y_{r}\right)$ of complex variables $y_{1}, \ldots, y_{r}(r \in \mathrm{N})$, let

$$
\begin{aligned}
& \Lambda_{\mu, p, q}\left(x_{1}, \ldots, x_{s} ; \beta, c_{1}, \ldots, c_{s} ; y_{1}, \ldots, y_{r} ; t\right) \\
& =\sum_{n=0}^{\infty} a_{n} m_{m+q n}\left(x_{1}, \ldots, x_{s} ; \beta, c_{1}, \ldots, c_{s}\right) \Omega_{\mu+p n}\left(y_{1}, \ldots, y_{r}\right) t^{n} \quad\left(a_{n} \neq 0, \mu \in \mathrm{C}\right) .
\end{aligned}
$$

Suppose also that

$$
\theta_{n, p, q}\left(y_{1}, \ldots, y_{r} ; z\right)=\sum_{k=0}^{[n / q]} a_{k} \Omega_{\mu+p k}\left(y_{1}, \ldots, y_{r}\right) \frac{z^{k}}{(n-q k) !}(\mu, p \in \mathrm{C})
$$

Then, for $p, q \in \mathrm{N}$, we have

$$
\begin{aligned}
& \sum_{n=0}^{\infty} m_{m+n}\left(x_{1}, \ldots, x_{s} ; \beta, c_{1}, \ldots, c_{s}\right) \theta_{n, p, q}\left(y_{1}, \ldots, y_{r} ; z\right) t^{n} \\
& =(1-t)^{-\beta-m-x_{1}-x_{2}-\ldots-x_{s}} \prod_{j=1}^{s}\left(1-\frac{t}{c_{j}}\right)^{x_{j}} \Lambda_{\mu, p, q}\left(x_{1}, \ldots, x_{s} ; \beta, \frac{c_{1}-t}{1-t}, \ldots, \frac{c_{s}-t}{1-t} ; y_{1}, \ldots, y_{r} ; z\left(\frac{t}{1-t}\right)^{q}\right) .
\end{aligned}
$$

Proof: Let $S$ denote the first member of the assertion (7) of Theorem 4. Then,

$$
S=\sum_{n=0}^{\infty} m_{m+n}\left(x_{1}, \ldots, x_{s} ; \beta, c_{1}, \ldots, c_{s}\right) \sum_{k=0}^{[n / q]} a_{k} \Omega_{\mu+p k}\left(y_{1}, \ldots, y_{r}\right) z^{k} \frac{t^{n}}{(n-q k) !} .
$$

Now, setting $n$ by $n+q k$ and then using relation (4), 


$$
\begin{gathered}
S=\sum_{n=0}^{\infty} \sum_{k=0}^{\infty} m_{m+n+q k}\left(x_{1}, \ldots, x_{s} ; \beta, c_{1}, \ldots, c_{s}\right) a_{k} \Omega_{\mu+p k}\left(y_{1}, \ldots, y_{r}\right) z^{k} \frac{t^{n+q k}}{n !} \\
=\sum_{k=0}^{\infty}\left(\sum_{n=0}^{\infty} m_{m+n+q k}\left(x_{1}, \ldots, x_{s} ; \beta, c_{1}, \ldots, c_{s}\right) \frac{t^{n}}{n !}\right) a_{k} \Omega_{\mu+p k}\left(y_{1}, \ldots, y_{r}\right)\left(z t^{q}\right)^{k} \\
=\sum_{k=0}^{\infty}(1-t)^{-\beta-m-q k-x_{1}-\ldots-x_{s}} \prod_{j=1}^{s}\left(1-\frac{t}{c_{j}}\right)^{x_{j}} \\
\times m_{m+q k}\left(x_{1}, \ldots, x_{s} ; \beta, \frac{c_{1}-t}{1-t}, \ldots, \frac{c_{s}-t}{1-t}\right) a_{k} \Omega_{\mu+p k}\left(y_{1}, \ldots, y_{r}\right)\left(z t^{q}\right)^{k} \\
=(1-t)^{-\beta-m-x_{1}-\ldots-x_{s}} \prod_{j=1}^{s}\left(1-\frac{t}{c_{j}}\right)^{x_{j}} \\
\times \Lambda_{\mu, p, q}\left(x_{1}, \ldots, x_{s} ; \beta, \frac{c_{1}-t}{1-t}, \ldots, \frac{c_{s}-t}{1-t} ; y_{1}, \ldots, y_{r} ; z\left(\frac{t}{1-t}\right)^{q}\right)
\end{gathered}
$$

which completes the proof.

\section{The Generalized Lauricella Functions}

In the present section, we derive various families of bilateral generating functions for the Meixner polynomials in several variables and the Lauricella functions. Some of the definitions and notations used in this paper are presented here as follows. A further generalization of the familiar Kampé de Fériet hypergeometric function in two variables is due to Srivastava and Daoust [8] who defined the Srivastava-Daoust (or generalized Lauricella) function as follows:

$$
\begin{aligned}
& F_{C: D^{(1)} ; \ldots ; D^{(n)}}^{A: B^{(1)}, \ldots B^{(n)}}\left(\begin{array}{llll}
{\left[(a): \theta^{(1)}, \ldots, \theta^{(n)}\right]:} & {\left[\left(b^{(1)}\right): \phi^{(1)}\right], \ldots ;} & {\left[\left(b^{(n)}\right): \phi^{(n)}\right] ;} & \\
{\left[(c): \psi^{(1)}, \ldots, \psi^{(n)}\right]:} & {\left[\left(d^{(1)}\right): \delta^{(1)}\right] \ldots ;} & {\left[\left(d^{(n)}\right): \delta^{(n)}\right]} & z_{1}, \ldots, z_{n}
\end{array}\right) \\
& =\sum_{m_{1}, \ldots, m_{n}=0}^{\infty} \Omega\left(m_{1}, \ldots, m_{n}\right) \frac{z_{1}^{m_{1}}}{m_{1} !} \ldots \frac{z_{n}^{m_{n}}}{m_{n} !},
\end{aligned}
$$

where for convenience

$$
\begin{aligned}
& \Omega\left(m_{1}, \ldots, m_{n}\right) \\
& :=\frac{\prod_{j=1}^{A}\left(a_{j}\right)_{m_{1} \theta_{j}^{(1)}+\ldots+m_{n} \theta_{j}^{(n)}}}{\prod_{j=1}^{C}\left(c_{j}\right)_{m_{1} \psi_{j}^{(1)}+\ldots+m_{n} \psi_{j}^{(n)}}} \frac{\prod_{j=1}^{B^{(1)}}\left(b_{j}^{(1)}\right)_{m_{1} \phi_{j}^{(1)}}}{\prod_{j=1}^{(1)}\left(d_{j}^{(1)}\right)_{m_{1} \delta_{j}^{(1)}}} \ldots \frac{\prod_{j=1}^{B^{(n)}}\left(b_{j}^{(n)}\right)_{m_{n} \phi_{j}^{(n)}}}{\prod_{j=1}^{(n)}\left(d_{j}^{(n)}\right)_{m_{n} \delta_{j}^{(n)}}}
\end{aligned}
$$

the coefficients

$$
\begin{aligned}
& \theta_{j}^{(k)}(j=1, \ldots, A ; k=1, \ldots, n), \text { and } \phi_{j}^{(k)}\left(j=1, \ldots, B^{(k)} ; k=1, \ldots, n\right), \\
& \psi_{j}^{(k)}(j=1, \ldots, C ; k=1, \ldots, n), \text { and } \delta_{j}^{(k)}\left(j=1, \ldots, D^{(k)} ; k=1, \ldots, n\right)
\end{aligned}
$$


are real constants and $\left(b_{B^{(k)}}^{(k)}\right)$ abbreviates the array of $B^{(k)}$ parameters $b_{j}^{(k)}\left(j=1, \ldots, B^{(k)} ; k=1, \ldots, n\right)$.

For a suitably bounded non-vanishing multiple sequence $\left\{\Omega\left(m_{1}, \ldots, m_{n}\right)\right\}_{m_{1}, \ldots, m_{n} \in \mathrm{N}_{0}}$ of real or complex parameters, we define a function $\phi_{n}\left(u_{1} ; u_{2}, \ldots, u_{s}\right)$ of $s$ variables $u_{1} ; u_{2}, \ldots, u_{s}$ (real or complex) be defined by [9],

$$
\begin{aligned}
& \phi_{n}\left(u_{1} ; u_{2}, \ldots, u_{s}\right) \\
& :=\sum_{m_{1}=0}^{n} \sum_{m_{2}, \ldots, m_{s}=0}^{\infty} \frac{(-n)_{m_{1}}((b))_{m_{1} \phi}}{((d))_{m_{1}} \delta} \Omega\left(f\left(m_{1}, \ldots, m_{s}\right), m_{2}, \ldots, m_{s}\right) \frac{u_{1}^{m_{1}}}{m_{1} !} \ldots \frac{u_{s}^{m_{s}}}{m_{s} !}
\end{aligned}
$$

where, for convenience

$$
((b))_{m_{1} \phi}=\prod_{j=1}^{B}\left(b_{j}\right)_{m_{1} \phi_{j}}, \quad((d))_{m_{1} \delta}=\prod_{j=1}^{D}\left(d_{j}\right)_{m_{1} \delta_{j}} .
$$

Theorem 5: The following bilateral generating function holds true:

$$
\begin{aligned}
& \sum_{n=0}^{\infty} m_{n}\left(x_{1}, \ldots, x_{s} ; \beta, c_{1}, \ldots, c_{s}\right) \phi_{n}\left(u_{1} ; u_{2}, \ldots, u_{r}\right) \frac{t^{n}}{n !} \\
& =(1-t)^{-\beta-x_{1}-\ldots-x_{s}} \prod_{j=1}^{s}\left(1-\frac{t}{c_{j}}\right)^{x_{j}} \\
& \times \sum_{m_{1}, k_{1}, \ldots, k_{s}, m_{2}, \ldots, m_{r}=0}^{\infty} \Omega\left(f\left(m_{1}+k_{1}+\ldots+k_{s}, \ldots, m_{r}\right), m_{2}, \ldots, m_{r}\right) \\
& \times \frac{((b))_{\left(m_{1}+k_{1}+\ldots+k_{s}\right) \phi}\left(\beta+k_{1}+\ldots+k_{s}\right)_{m_{1}}\left(-x_{1}\right)_{k_{1}} \ldots\left(-x_{s}\right)_{k_{s}}}{((d))_{\left(m_{1}+k_{1}+\ldots+k_{s}\right) \delta}} \\
& \times \frac{\left(\frac{u_{1} t}{t-1}\right)^{m_{1}}}{m_{1} !} \frac{\left(\frac{u_{1} t}{1-t}\left(\frac{c_{1}-1}{c_{1}-t}\right)\right)^{k_{1}}}{k_{1} !} \ldots \frac{\left(\frac{u_{1} t}{1-t}\left(\frac{c_{s}-1}{c_{s}-t}\right)\right)^{k_{s}}}{k_{s} !} \frac{u_{2}}{m_{2} !} \ldots \frac{u_{r}}{m_{r} !},
\end{aligned}
$$

where $\phi_{n}\left(u_{1} ; u_{2}, \ldots, u_{r}\right)$ is given by (4).

Proof: By using the relationship (4), we can easily derive the following generating functions

$$
\begin{gathered}
\sum_{n=0}^{\infty} m_{n}\left(x_{1}, \ldots, x_{s} ; \beta, c_{1}, \ldots, c_{s}\right) \phi_{n}\left(u_{1} ; u_{2}, \ldots, u_{r}\right) \frac{t^{n}}{n !} \\
=\sum_{n=0}^{\infty} m_{n}\left(x_{1}, \ldots, x_{s} ; \beta, c_{1}, \ldots, c_{s}\right)\left(\sum_{m_{1}=0}^{n} \sum_{m_{2}, \ldots, m_{r}=0}^{\infty} \frac{(-n)_{m_{1}}((b))_{m_{1} \phi}}{((d))_{m_{1} \delta}}\right.
\end{gathered}
$$




$$
\begin{gathered}
\left.\times \Omega\left(f\left(m_{1}, \ldots, m_{r}\right), m_{2}, \ldots, m_{r}\right) \frac{u_{1}^{m_{1}}}{m_{1} !} \ldots \frac{u_{r}^{m_{r}}}{m_{r} !}\right) \frac{t^{n}}{n !} \\
=(1-t)^{-\beta-x_{1}-\ldots-x_{s}} \prod_{j=1}^{s}\left(1-\frac{t}{c_{j}}\right)^{x_{j}} \\
\times \sum_{m_{1}, k_{1}, \ldots, k_{s}, m_{2}, \ldots, m_{r}=0}^{\infty} \frac{((b))_{\left(m_{1}+k_{1}+\ldots+k_{s}\right) \phi}\left(\beta+k_{1}+\ldots+k_{s}\right)_{m_{1}}\left(-x_{1}\right)_{k_{1}} \ldots\left(-x_{s}\right)_{k_{s}}}{((d))_{\left(m_{1}+k_{1}+\ldots+k_{s}\right) \delta}} \\
\times \Omega\left(f\left(m_{1}+k_{1}+\ldots+k_{s}, m_{2}, \ldots, m_{r}\right), m_{2}, \ldots, m_{r}\right) \\
\times \frac{\left(\frac{u_{t} t}{t-1}\right)^{m_{1}}}{m_{1} !} \frac{\left(\frac{u_{1} t}{1-t}\left(\frac{c_{1}-1}{c_{1}-t}\right)\right)^{k_{1}}}{k_{1} !} \ldots \frac{\left(\frac{u_{1} t}{1-t}\left(\frac{c_{s}-1}{c_{s}-t}\right)\right)^{k_{s}}}{k_{s} !} \frac{u_{2}^{m_{2}}}{m_{2} !} \ldots \frac{u_{r}^{m_{r}}}{m_{r} !} .
\end{gathered}
$$

Corollary 1: Upon setting

$$
\Omega\left(f\left(m_{1}, \ldots, m_{r}\right), m_{2}, \ldots, m_{r}\right)=\frac{(a)_{m_{1}+\ldots+m_{r}}\left(b_{2}\right)_{m_{2}} \ldots\left(b_{r}\right)_{m_{r}}}{\left(c_{1}\right)_{m_{1}} \ldots\left(c_{r}\right)_{m_{r}}}
$$

and $\phi=\delta=0$ in Theorem 5,

$$
\begin{gathered}
\sum_{n=0}^{\infty} m_{n}\left(x_{1}, \ldots, x_{s} ; \beta, c_{1}, \ldots, c_{s}\right) F_{A}^{(r)}\left[a,-n, b_{2}, \ldots, b_{r} ; c_{1}, \ldots, c_{r} ; u_{1}, \ldots, u_{r}\right] \frac{t^{n}}{n !} \\
=(1-t)^{-\beta-x_{1}-\ldots-x_{s}} \prod_{j=1}^{s}\left(1-\frac{t}{c_{j}}\right)^{x_{j}} F_{1: 0 ; 0 ; \ldots ; 0 ; 1 ; \ldots ; 1}^{1: 1 ; 1 ; \ldots ; 1 ; 1 ; ; 1} \\
\left(\begin{array}{c}
{[(a): 1, \ldots, 1]: \quad\left[\beta+k_{1}+\ldots+k_{s}: 1\right] ;\left[-x_{1}: 1\right] ; \ldots ;\left[-x_{s}: 1\right] ;} \\
{\left[\left(c_{1}\right): \psi^{(1)}, \ldots, \psi^{(s+r)}\right]:}
\end{array}\right. \\
{\left[\left(b^{(2)}\right): \phi^{(2)}\right] ; \quad \ldots ; \quad\left[\left(b^{(r)}\right): \phi^{(r)}\right] ;\left(\frac{u_{1} t}{t-1}\right)\left(\frac{u_{1} t}{1-t}\left(\frac{c_{1}-1}{c_{1}-t}\right)\right) \ldots,\left(\frac{u_{1} t}{1-t}\left(\frac{c_{s}-1}{c_{S}-t}\right)\right), u_{2}, \ldots, u_{r}}
\end{gathered}
$$

where $F_{A}^{(s)}$ the Lauricella function and the coefficients $\psi^{(\eta)}$ are given by

$$
\psi^{(\eta)}=\left\{\begin{array}{cc}
1 & (1 \leq \eta \leq s+1) \\
0 & (s+1<\eta \leq s+r)
\end{array} .\right.
$$

Corollary 2: If we put

$$
\Omega\left(f\left(m_{1}, \ldots, m_{r}\right), m_{2}, \ldots, m_{r}\right)=\frac{\left(a_{1}^{(1)}\right)_{m_{2}} \ldots\left(a_{1}^{(r-1)}\right)_{m_{r}}\left(a_{2}^{(1)}\right)_{m_{2}} \ldots\left(a_{2}^{(r-1)}\right)_{m_{r}}}{(c)_{m_{1}+\cdots+m_{r}}}
$$

and $\phi_{1}=1, B=1, \delta=0$ and $b_{1}=b$ in Theorem 5, 


$$
\begin{aligned}
& \sum_{n=0}^{\infty} m_{n}\left(x_{1}, \ldots, x_{s} ; \beta, c_{1}, \ldots, c_{s}\right) F_{B}^{(r)}\left[-n, a_{1}^{(1)}, \ldots, a_{1}^{(r-1)}, b, a_{2}^{(1)}, \ldots, a_{2}^{(r-1)} ; c ; u_{1}, \ldots, u_{r}\right] \frac{t^{n}}{n !} \\
& =(1-t)^{-\beta-x_{1}-\ldots-x_{s}} \prod_{j=1}^{s}\left(1-\frac{t}{c_{j}}\right)^{x_{j}} F_{1: 0 ; 0 ; \ldots ; 0 ; 0 ; \ldots ; 0}^{1: 1 ; 1 ; \ldots ; 1 ; ; \ldots ;} \\
& \left(\left[(b): \theta^{(1)}, \ldots, \theta^{(s+r)}\right]:\left[\beta+k_{1}+\ldots+k_{s}: 1\right] ;\left[-x_{1}: 1\right] ; \ldots ;\left[-x_{s}: 1\right]\right. \text {; } \\
& {\left[\begin{array}{lllll}
c & (6): 11]
\end{array}\right] \quad-\quad \ldots ; \quad-;}
\end{aligned}
$$

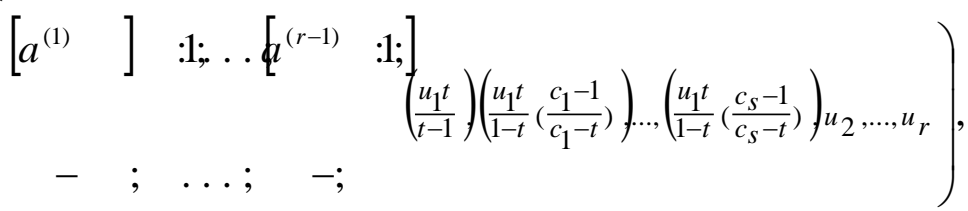

where $F_{B}^{(s)}$ the Lauricella function and the coefficients $\theta^{(\eta)}$ are given by

$$
\theta^{(\eta)}=\left\{\begin{array}{cc}
1, & 1 \leq \eta \leq s+1 \\
0, & s+1<\eta \leq s+r
\end{array}\right.
$$

Corollary 3: Finallay, if we let

$$
\Omega\left(f\left(m_{1}, \ldots, m_{r}\right), m_{2}, \ldots, m_{r}\right)=\frac{(a)_{m_{1}+\ldots+m_{r}}\left(b_{2}\right)_{m_{2}} \ldots\left(b_{r}\right)_{m_{r}}}{(c)_{m_{1}+\ldots+m_{r}}}
$$

and $\phi=\delta=0$ in Theorem 5, we have the following result:

$$
\begin{gathered}
\sum_{n=0}^{\infty} m_{n}\left(x_{1}, \ldots, x_{s} ; \beta, c_{1}, \ldots, c_{s}\right) F_{D}^{(r)}\left[a,-n, b_{2}, \ldots, b_{r} ; c ; u_{1}, u_{2}, \ldots, u_{r}\right] \frac{t^{n}}{n !} \\
=(1-t)^{-\beta-x_{1}-\ldots-x_{s}} \prod_{j=1}^{s}\left(1-\frac{t}{c_{j}}\right)^{x_{j}} F_{D}^{(s+r)}\left[a, \beta+k_{1}+\ldots+k_{s},-x_{1}, \ldots,-x_{s}, b_{2}, \ldots, b_{r} ; c ;\right. \\
\left.\left(\frac{u_{1} t}{t-1}\right),\left(\frac{u_{1} t}{1-t}\left(\frac{c_{1}-1}{c_{1}-t}\right)\right), \ldots,\left(\frac{u_{1} t}{1-t}\left(\frac{c_{s}-1}{c_{s}-t}\right)\right), u_{2}, \ldots, u_{r}\right] .
\end{gathered}
$$

\section{References}

[1] Chihara, T. S. (1978). An Introduction to Orthogonal Polynomials. New York: Gordon and Breach Science Publishers Inc.

[2] Khan, M. A., \& Akhlaq, M. (2012). A note on generating functions and summation formulae for Meixner polynomials of several variables. Demonstratio Mathematica, 1, 51-66.

[3] Chen, K.-Y., \& Srivastava, H. M. (2005). Series identities and associated families of generating functions. J. Math. Anal. Appl., 311, 582-599.

[4] Erkus-Duman, E., Altın, A., \& Aktas, R. (2011). Miscellaneous properties of some multivariable polynomials. Math. Comput. Modelling, 54, 1875-1885.

[5] Özmen, N., \& Erkus-Duman, E. (2018). Some families of generating functions for the generalized Cesáro 
polynomials. J. Comput. Anal. Appl., 25, 670-683.

[6] Korkmaz-Duzgun, D., \& Erkus-Duman, E. (2018). The laguerre type $d$-orthogonal polynomials. J. Sci. Arts, 42, 95-106.

[7] Özmen, N. (2017). Some new properties of the Meixner polynomials. Sakarya University Journal of Science, 21(6), 1454-1462.

[8] Srivastava, H. M., \& Daoust, M. C. (1969). Certain generalized Neumann expansions associated with the Kampé de Fériet function. Nederl. akad. Westensch. Indag. Math, 31, 449-457.

[9] Liu, S.-J., Lin, S.-D., Srivastava, H. M., \& Wong, M.-M. (2012). Bilateral generating functions for the Erkuş-Srivastava polynomials and the generalized Lauricella functions. App. Mathematcis and Comp., 218, 7685-7693.

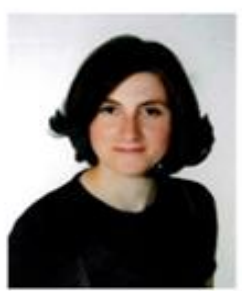

Nejla Özmen is an assistant professor of mathematics at Düzce University in Düzce, Turkey. In 2012 she received the Ph.D. degree from the Department of Mathematics in Gazi University. She has been working in Düzce University since 2009. Her research areas contain special functions, generating functions.

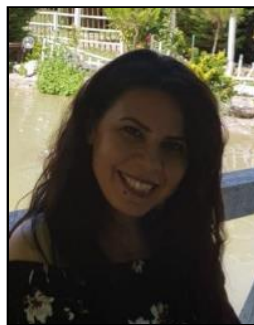

Esra Erkus-Duman is a full professor of mathematics at Gazi University in Ankara, Turkey. In 2005 she received the Ph.D. degree from the Department of Mathematics in Ankara University. Before she came to Gazi University, she worked as a research assistant at Ankara University. She became associate professor in 2010 and full professor in 2017. Her research areas contain special functions, generating functions and orthogonal polynomials. 